\title{
An efficient stereoselective total synthesis of 11ß-methoxycurvularin
}

\author{
J. S. Yadav, * C. Divya Vani, N. Bhasker, and B. V. Subba Reddy \\ Natural Products Chemistry Division, Indian Institute of Chemical Technology, \\ Hyderabad 500 007, India \\ E-mail:yadavpub@iict.res.in
}

DOI: http://dx.doi.org/10.3998/ark.5550190.p008.453

\begin{abstract}
A very short and efficient stereoselective total synthesis of a macrocyclic ketone, 11 $\beta$-methoxycurvularin was achieved by employing the Sharpless asymmetric epoxidation, formation of propargyl alcohols from an epoxy-chloride, and intramolecular Friedel-Crafts acylation as the key steps.
\end{abstract}

Keywords: Sharpless epoxidation, epoxy chloride, propargyl alcohol, intramolecular FriedelCrafts acylation

\section{Introduction}

11ß-Methoxycurvularin (5) was first isolated from the mycelium of hybrid strain ME-005 which is a polyketide metabolite of various Curvularia, Pencillium, Alternaria, and Cochiobolous species. It was found to exhibit cytotoxicity ${ }^{1}$ and antimicrobial activities ${ }^{2.3}$ against four types of human cancer cell lines such as [NCI-H460, MCF-7, SF-268, 41A Pa Ca-2]. ${ }^{4}$ It also shows some effect in the spindle formation of embryos of sea urchin cells to give barrel like spindles and terminate the first step of cell division, which is a promising tool for anticancer drug discovery. Furthermore, it also shows binding affinity with tubulins. ${ }^{5}$

Structurally, 11ß-methoxycurvularin shows different configuration at C-11 in the 12membered lactone ring. The first total synthesis of these natural products has been reported by Liang et $a l^{6}{ }^{6}$ which led to a revision of the spectroscopic data of the originally proposed structures (4 and 5). 
<smiles>CCCCC(=O)c1c(O)cc(O)cc1CC(=O)OC(C)C</smiles><smiles></smiles><smiles>C[C@H]1CCC[C@@H](O)CC(=O)c2c(O)cc(O)cc2CC(=O)O1</smiles><smiles>CO[C@H]1CCC[C@@H](C)OC(=O)Cc2cc(O)cc(O)c2C(=O)C1</smiles><smiles>CO[C@H]1CCC[C@@H](C)OC(=O)Cc2cc(O)cc(O)c2C(=O)C1</smiles>

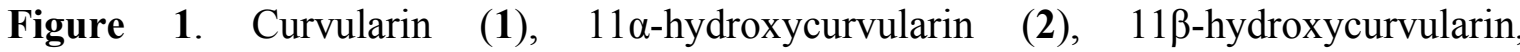
(3), $11 \alpha$-methoxycurvularin (4), 11 $\beta$-methoxycurvularin (5).

We herein report a new synthetic route tor the stereoselective total synthesis of $11(\beta)$ methoxycurvularin. Our approach utilizes mainly the Sharpless asymmetric epoxidation to introduce absolute stereocentre at $\mathrm{C}-11$ for the construction of the key fragment $\mathbf{8}$, which in turn comes from a commercially available homopropargyl alcohol 12.<smiles>C#CCCOc1ccccc1</smiles>

Scheme1. Retrosynthetic strategy to $11 \beta$-methoxycurvularin (5).

Accordingly, the aromatic portion was expected to arise from the readily available 3,5dimethoxyphenylacetic acid $7^{7,8}$ Retrosynthesis of the 12-membered lactone ring 5 led to a known compound $\mathbf{6}$ along with the key fragment $\mathbf{8}$, which in turn could be prepared from 9 and 10 in 65\% yield. The fragment 9 was synthesized from homopropargyl alcohol 12 in seven steps. 
Thus treatment of homopropargyl alcohol 12 with $\mathrm{BnBr}$ in the presence of $\mathrm{NaH}^{9,10}$ gave the benzyl ether 13 (Scheme 2) which upon reaction with paraformaldehyde in the presence of magnesium turnings and ethyl bromide gave the alcohol 14 in $72 \%$ yield. Reduction of 14 with LAH furnished an allyl alcohol 15, which was then subjected to the Sharpless asymmetric epoxidation $^{11,12}$ by using titanium isopropoxide, L(+)-DIPT, 4.5 M TBHP to afford the epoxide with 95\% ee. This chiral epoxide was then converted into propargyl alcohol 17 which was subsequently protected as its methyl ether 9 by using $\mathrm{NaH}$ and $\mathrm{MeI}$ in $85 \%$ yield. Regioselective ring opening of $(S)$-methyloxirane 10 with 9 using $n-\mathrm{BuLi}, \mathrm{BF}_{3} \mathrm{OEt}_{2}$ gave the secondary homopropargyl alcohol 8 in $65 \%$ overall yield. ${ }^{13}$

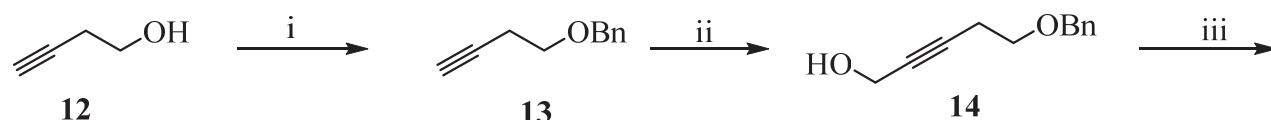

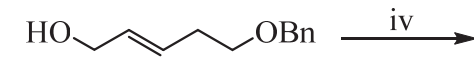

15
13
14

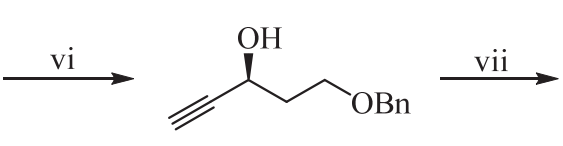

17

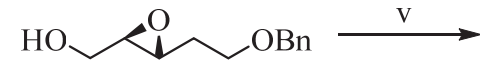

11

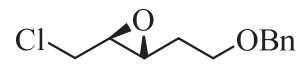

16

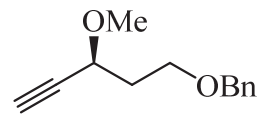

9

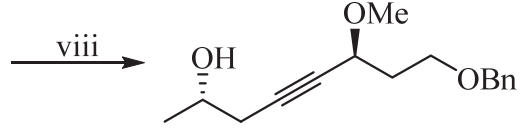

8

Reagents and conditions: i) $\mathrm{BnBr}, \mathrm{NaH}$, dry $\mathrm{THF}, 0^{\circ}$-rt, 93\%; ii) EtMgBr, $\mathrm{Mg}$ turnings, $(\mathrm{HCHO})_{\mathrm{n}}$, dry THF 72\%; iii) LAH, dry THF, reflux, 90\%; iv) $4 \AA \mathrm{MS}, \mathrm{L}(+)$-DIPT, Ti(OiPr) 4 , 4.5 M TBHP, DCM, $27{ }^{\circ} \mathrm{C}, 70 \%$; v) TPP, $\mathrm{NaHCO}_{3}, \mathrm{CCl}_{4}$, reflux, 80\%; vi) Li, liq $\mathrm{NH}_{3}$, $\mathrm{Fe}\left(\mathrm{NO}_{3}\right)_{3},-33{ }^{\circ} \mathrm{C}, 70 \%$; vii) $\mathrm{NaH}$, MeI, dry THF, $85 \%$; viii) methyloxirane, $n$-BuLi, $-78{ }^{\circ} \mathrm{C}$, dry THF, 65\%.

Scheme 2. Synthesis of the ynol 8.

As shown in the Scheme 3, the esterification ${ }^{7}$ of $\mathbf{8}$ with 3,5-dimethoxyphenylacetic acid 7 using DCC and DMAP at room temperature afforded compound 18 in $80 \%$ yield. Subsequent deprotection of the benzyl group and reduction of the triple bond were achieved by hydrogenation using $10 \% \mathrm{Pd} / \mathrm{C}^{14}$ in ethyl acetate to afford the alcohol 19 in $70 \%$ yield. Oxidation of 19 using Jones reagent ${ }^{15,16}\left(\mathrm{CrO}_{3}, \mathrm{H}_{2} \mathrm{O}, \mathrm{H}_{2} \mathrm{SO}_{4}\right.$; acetone, $0^{\circ}$-rt $)$ gave the desired carboxylic acid 6 in $80 \%$ yield.

Intramolecular Friedel-Crafts acylation of $\mathbf{6}$ was achieved using a mixture of trifluoroacetic acid and trifluoroacetic anhydride ${ }^{17-20}$ to give a macrolide $\mathbf{2 0}$ in $50 \%$ yield. Finally, the deprotection of $\mathbf{2 0}$ with freshly prepared $\mathrm{AlI}_{3}$ in benzene gave the target molecule, $11 \beta$ methoxycurvularin 5 in $65 \%$ yield as colorless oil. 


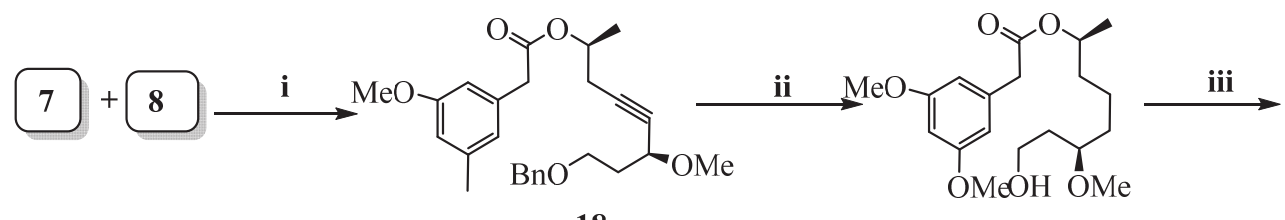

18

19

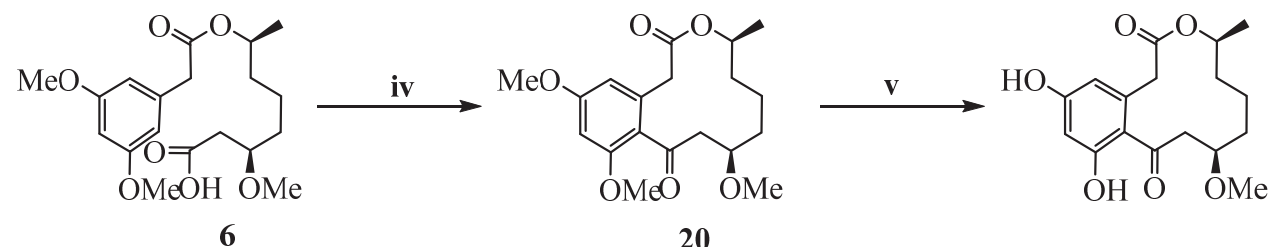

5

Reagents and conditions: (i) DCC, DMAP, DCM, 80\%; (ii) 10\% Pd/C, EtOAc, 70\%; (iii) $\mathrm{CrO}_{3}$, acetone, $\mathrm{H}_{2} \mathrm{SO}_{4}, 80 \%$; (iv) TFA, TFAA, $50 \%$; (v) $\mathrm{AlI}_{3}$, TBAI, benzene, $15{ }^{\circ} \mathrm{C}, 65 \%$.

\section{Scheme 3}

\section{Conclusions}

In summary, we have successfully demonstrated an efficient total synthesis of $11 \beta$ methoxycurvularin in a highly stereoselective manner. The synthesis utilizes the Sharpless asymmetric epoxidation, and an intramolecular Friedel-crafts acylation to construct 12membered macrocyclic lactone ring system.

\section{Experimental Section}

General. The reactions were conducted under $\mathrm{N}_{2}$ atmosphere using anhydrous solvents such as DCM, THF. All reactions were monitored by thin layer chromatography (TLC) using silicacoated plates and visualizing under UV light. Yields refer to chromatographically pure products by ${ }^{1} \mathrm{H}$ and ${ }^{13} \mathrm{C}$ NMR. Air sensitive reagents were transferred by a syringe or with a double-ended needle. Evaporation of the solvent was performed at reduced pressure using a Buchi rotary evaporator. ${ }^{1} \mathrm{H}$ NMR spectra were recorded on Varian FT-200MHz (Gemini) and Bruker UXNMR FT-300MHz (Avance) in $\mathrm{CDCl}_{3}$. Chemical shift values were reported in ppm relative to tetramethylsilane $(\delta 0.0)$ as an internal standard. Mass spectra were recorded under electron impact at $70 \mathrm{eV}$ on LC-MS (Agilent Technologies). Column chromatography was performed on silica gel (60-120 mesh) supplied by Acme Chemical Co., India. Thin-layer chromatography was performed on Merck 60 F-254 silica gel plates. Optical rotations were measured with JASCO DIP-370 polarimeter. 
[(But-3-ynyloxy)methyl]benzene (13). To a suspension of $\mathrm{NaH}$ (1.13 g, $27.85 \mathrm{mmol})$ in THF $(50 \mathrm{~mL})$, was added alcohol $12(1.5 \mathrm{~g}, 21.42 \mathrm{mmol})$ at $0{ }^{\circ} \mathrm{C}$. After stirring for $20 \mathrm{~min}, \mathrm{BnBr}(3.66$ $\mathrm{g}, 21.42 \mathrm{mmol}$ ) was added slowly and the resulting mixture was stirred for another $4 \mathrm{~h}$. After completion, the reaction was quenched with ice-cold $\mathrm{H}_{2} \mathrm{O}(20 \mathrm{~mL})$ and extracted with EtOAc $(2 \times 50 \mathrm{~mL})$. The combined organic layers were dried over anhydrous $\mathrm{Na}_{2} \mathrm{SO}_{4}$. The solvent was evaporated under reduced pressure and the residue was purified by column chromatography (EtOAc/hexanes 1:9) to afford compound $\mathbf{1 3}$ as a yellow oily liquid (3.1 g, 93\%).

IR (neat) $v_{\max }: 3031,2916,2865,2120,1454,1363,1204,1028,820,739,698 \mathrm{~cm}^{-1}$. ${ }^{1} \mathrm{H}$ NMR $\left(\mathrm{CDCl}_{3}, 500 \mathrm{MHz}\right): \delta 7.21-7.31(\mathrm{~m}, 5 \mathrm{H}), 4.52(\mathrm{~s}, 2 \mathrm{H}), 3.56(\mathrm{t}, J 6.8 \mathrm{~Hz}, 2 \mathrm{H}), 2.46(\mathrm{td}, J 2.9,6.8$ $\mathrm{Hz}, 2 \mathrm{H}), 1.88$ (t, $J 2.9 \mathrm{~Hz}, 1 \mathrm{H})$. EI-MS: $m / z: 159(\mathrm{M}-\mathrm{H})$.

5-(Benzyloxy)pent-2-yn-1-ol (14). To a suspension of $\mathrm{Mg}$ metal (2.5 g, $15.62 \mathrm{mmol})$ in dry THF $(50 \mathrm{~mL})$ was added ethyl bromide $(1.7 \mathrm{~mL}, 23.43 \mathrm{mmol})$ at $0{ }^{\circ} \mathrm{C}$. The mixture was stirred for 30 min at room temperature and then compound $\mathbf{1 3}$ in dry THF was added at $0{ }^{\circ} \mathrm{C}$. After stirring for $2 \mathrm{~h}$ at room temperature, paraformaldehyde was added slowly at $0{ }^{\circ} \mathrm{C}$. The resulting mixture was stirred for another 5-6 h. After completion, the reaction was quenched with saturated $\mathrm{NH}_{4} \mathrm{Cl}(20$ $\mathrm{mL})$ and extracted with EtOAc $(2 \times 50 \mathrm{~mL})$ and dried over $\mathrm{Na}_{2} \mathrm{SO}_{4}$. The solvent was evaporated under reduced pressure and the residue was purified by column chromatography (EtOAc/hexanes, 2:8) as eluent to afford compound $\mathbf{1 4}$ as a colourless oily liquid (1.65 $\mathrm{g}$ (72\%). IR (neat) $v_{\max }: 3064,3031,2289,2237,1958,1718,1417,1363,1273,1137,1014,744,699$ $\mathrm{cm}^{-1} .{ }^{1} \mathrm{H}$ NMR $\left(\mathrm{CDCl}_{3}, 300 \mathrm{MHz}\right): \delta 7.20-7.35(\mathrm{~m}, 5 \mathrm{H}), 4.52(\mathrm{~s}, 2 \mathrm{H}), 4.16(\mathrm{~s}, 2 \mathrm{H}), 3.54(\mathrm{t}, J 6.8$ $\mathrm{Hz}, 2 \mathrm{H}), 2.49$ (t, J $6.8 \mathrm{~Hz}, 2 \mathrm{H}), 1.89-195$ (bs, $1 \mathrm{H}) .{ }^{13} \mathrm{C} \mathrm{NMR}\left(\mathrm{CDCl}_{3}, 75 \mathrm{MHz}\right): \delta 137.3,127.8$, 127.2, 127.1, 81.7, 79.4, 72.2, 67.7, 50.0, 19.5. EI-MS: $m / z 189$ [M-H].

(E)-5-(Benzyloxy)pent-2-en-1-ol (15). To a suspension of $\mathrm{LiAlH}_{4}(540 \mathrm{mg}, 14.21 \mathrm{mmol})$ in THF (30 mL) was added a solution of $14(1.8 \mathrm{~g}, 9.47 \mathrm{mmol})$ in THF $(10 \mathrm{~mL})$ in a dropwise manner. After stirring the mixture for $2 \mathrm{~h}$ at reflux, water was added. The resulting mixture was filtered through a short pad of Celite and washed with EtOAc $(3 \times 50 \mathrm{~mL})$. The filtrate was dried over anhydrous $\mathrm{Na}_{2} \mathrm{SO}_{4}$. The solvent was removed and the residue was purified on silica gel column using (EtOAc/hexanes 2:8) as the eluent to afford 15 as a colourless oil $(1.65 \mathrm{~g}$ (90\%) IR (neat) $v_{\max }: 2862,2359,1686,1453,1363,1309,1205,1098,971,910,739,698 \mathrm{~cm}^{-1} .{ }^{1} \mathrm{H}$ NMR: $\left(\mathrm{CDCl}_{3}, 300 \mathrm{MHz}\right): \delta 7.21-7.33(\mathrm{~m}, 5 \mathrm{H}), 5.68(\mathrm{td}, J 4.53,18.8 \mathrm{~Hz}, 2 \mathrm{H}), 4.48(\mathrm{~s}, 2 \mathrm{H}), 4.04$ $(\mathrm{d}, J 2.83 \mathrm{~Hz}, 2 \mathrm{H}), 3.52(\mathrm{t}, J 2.83 \mathrm{~Hz}, 2 \mathrm{H}), 2.34(\mathrm{td}, J 2.8,4.5 \mathrm{~Hz}, 2 \mathrm{H}) .{ }^{13} \mathrm{C} \mathrm{NMR}:\left(\mathrm{CDCl}_{3}, 75\right.$ MHz): $\delta 137.8,130.9,128.0,127.3,127.2,72.4,69.2,62.6,32.2$. EI-MS: $m / z: 191[\mathrm{M}-\mathrm{H}]$.

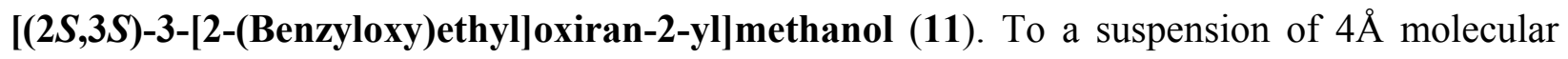
sieves $(1.5 \mathrm{~g})$ in $30 \mathrm{~mL} \mathrm{CH} \mathrm{Cl}_{2}$ were added L-(+)-diisopropyl tartrate $(0.18 \mathrm{~mL}, 0.782 \mathrm{mmol})$, $\mathrm{Ti}(\mathrm{O} i \mathrm{Pr})_{4}(0.22 \mathrm{~mL}, 0.782 \mathrm{mmol})$, and ${ }^{\mathrm{t}} \mathrm{BuOOH}(4.5 \mathrm{M}$ in toluene $(2.6 \mathrm{ml}, 11.71 \mathrm{mmol})$ sequentially at $-27{ }^{\circ} \mathrm{C}$. The mixture was stirred for $30 \mathrm{~min}$ and compound 15 (2.06 $\left.\mathrm{g}, 10 \mathrm{mmol}\right)$ was then added. The reaction mixture was stored overnight $(12 \mathrm{~h})$ in the freezer at $-25^{\circ} \mathrm{C}$ without stirring. The reaction was then warmed to $25{ }^{\circ} \mathrm{C}$ and quenched by the addition of $10 \%$ $\mathrm{NaOH} /$ saturated aqueous $\mathrm{NaCl}(4.0 \mathrm{~mL})$. Upon further warming to $-10{ }^{\circ} \mathrm{C}$ the mixture was diluted with $\mathrm{Et}_{2} \mathrm{O}(100 \mathrm{~mL})$ and then treated with $\mathrm{MgSO}_{4}(4.0 \mathrm{~g})$ and Celite $(1.0 \mathrm{~g})$ and was 
stirred for an additional $15 \mathrm{~min}$. The resulting mixture was then allowed to settle for $1 \mathrm{~h}$ before filtration through Celite using $\mathrm{Et}_{2} \mathrm{O}$ and then concentrated under reduced pressure. The residue was purified on silica gel column chromatography using (EtOAc/hexanes, 3:7) as eluent to afford compound $\mathbf{1 1}$ as colourless oil $(1.63 \mathrm{~g}, 70 \%)$.

$[\alpha]_{\mathrm{D}}^{27}+2.8\left(c, 0.5, \mathrm{CHCl}_{3}\right)$. IR (neat) $v_{\max }: 2927,2872,1453,1277,1098,1074,1027,750,716$, $699 \mathrm{~cm}^{-1} .{ }^{1} \mathrm{H}$ NMR $\left(\mathrm{CDCl}_{3}, 300 \mathrm{MHz}\right): \delta 7.21-7.32(\mathrm{~m}, 5 \mathrm{H}), 4.49(\mathrm{~s}, 2 \mathrm{H}), 3.20(\mathrm{dd}, J 2.2,10.5$ $\mathrm{Hz}, 1 \mathrm{H}), 3.50-3.59(\mathrm{~m}, 3 \mathrm{H}), 3.04(\mathrm{td}, J 2.2,6.7 \mathrm{~Hz}, 1 \mathrm{H}), 2.87-2.91(\mathrm{~m}, 1 \mathrm{H}), 2.34-2.41$ (bs, $1 \mathrm{H})$, 1.71-1.95 (m, 2H). ${ }^{13} \mathrm{C}$ NMR: $\left(\mathrm{CDCl}_{3}, 75 \mathrm{MHz}\right): \delta 137.6,127.8,127.5,127.3,123.9,125.9$, 72.4, 66.3, 61.3, 58.2, 53.2, 31.5, 31.0. ESI-MS: $m / z$ : 231 [M+Na]. HRMS calcd for $\mathrm{C}_{12} \mathrm{H}_{16} \mathrm{O}_{3} \mathrm{Na}$ 231.0997 , found 231.0996 .

(2S,3R)-2-[2-(Benzyloxy)ethyl]-3-(chloromethyl)oxirane (16). To a stirred solution of 11 (800 $\mathrm{mg}, 3.84 \mathrm{mmol})$ in anhydrous $\mathrm{CCl}_{4}(15 \mathrm{ml})$ was added triphenylphosphine $(102 \mathrm{~g}, 4.61 \mathrm{mmol})$ under nitrogen atmosphere and then sodium bicarbonate $(969 \mathrm{mg}, 11.5 \mathrm{mmol})$ was added. The mixture was heated under reflux until triphenylphosphine oxide separated from the reaction mixture. After $6 \mathrm{~h}$, the mixture was filtered and the solvent was removed from the filtrate under reduced pressure. The residue was purified by silica gel column chromatography (EtOAc / hexanes 1:9) to afford compound $\mathbf{1 6}$ as a colourless liquid. (691 $\mathrm{mg}, 80 \%$ ).

$[\alpha]_{\mathrm{D}}{ }^{27}+7.7\left(c, 0.5, \mathrm{CHCl}_{3}\right)$. IR (neat) $v_{\max }: 3031,2921,2852,1454,1360,1264,1028,878,736$, $698 \mathrm{~cm}^{-1} .{ }^{1} \mathrm{H}$ NMR: $\left(\mathrm{CDCl}_{3}, 300 \mathrm{MHz}\right): \delta$ 7.22-7.34 (m, 5H), $4.50(\mathrm{~s}, 2 \mathrm{H}), 3.56(\mathrm{tdd}, J$ 5.2, 6.0 , $11.3 \mathrm{~Hz}, 3 \mathrm{H}$ ), 3.44 (dd, $J 5.2,11.33 \mathrm{~Hz}, 1 \mathrm{H}$ ), 2.99 (td, $J 2.2,5.2,6.0 \mathrm{~Hz}, 2 \mathrm{H}), 1.73-1.96$ (m, $2 \mathrm{H})$. ${ }^{13} \mathrm{C} \mathrm{NMR}\left(\mathrm{CDCl}_{3}, 75 \mathrm{MHz}\right): 137.89,128.02,127.24,127.73,72.64,67.50,66.29,66.20,56.78$, 56.20, 44.49, 31.61. ESI-MS: $m / z$ : 251 [M+Na]. HRMS calcd for $\mathrm{C}_{12} \mathrm{H}_{15} \mathrm{O}_{2} \mathrm{Na} 249.0658$, found 249.0660 .

(S) 5-(Benzyloxy)pent-1-yn-3-ol (17). To freshly distilled ammonia $(25 \mathrm{~mL})$ in a $100 \mathrm{~mL}$ two neck round bottom flask fitted with a cold finger condenser, was added catalytic amount of ferric nitrite, followed by the piecewise addition of lithium metal $(166 \mathrm{mg}, 18.5 \mathrm{mmol})$ at $-33^{\circ} \mathrm{C}$. The resulting grey color suspension was stirred for $30 \mathrm{~min}$. To this was added the chloride $\mathbf{1 6}$ (600 $\mathrm{mg}, 2.64 \mathrm{mmol})$ in dry THF $(3 \mathrm{~mL})$ over a period of $5 \mathrm{~min}$. After stirring the reaction mixture for $30 \mathrm{~min}$, solid $\mathrm{NH}_{4} \mathrm{Cl}(2 \mathrm{~g})$ was added and ammonia was allowed to evaporate. The residue was partitioned between water and ether and the aqueous layer was extracted with ether $(2 \times 50 \mathrm{~mL})$. The combined organic layers were dried over anhydrous $\mathrm{Na}_{2} \mathrm{SO}_{4}$ and the solvent was evaporated under reduced pressure and the residue was purified by silica gel column chromatography (EtOAc/hexanes, 2.5:7.5) to afford compound 17 as a colourless liquid (346 mg, 70\%).

$[\alpha]_{\mathrm{D}}^{27}+4.5\left(c, 0.5, \mathrm{CHCl}_{3}\right)$. IR (neat) $v_{\max } 3410,3292,2926,2866,2113,1724,1601,1450$, 1364, 1206, 1084, 1023, 740, 696 $\mathrm{cm}^{-1} .{ }^{1} \mathrm{H}$ NMR: $\left(\mathrm{CDCl}_{3}, 300 \mathrm{MHz}\right): \delta 7.21-7.35$ (m, 5H), 4.46 $-4.66(\mathrm{~m}, 3 \mathrm{H}), 3.83(\mathrm{td}, J 4.5,9.8 \mathrm{~Hz}, 1 \mathrm{H}), 3.65(\mathrm{td}, J 4.5,9.8 \mathrm{~Hz}, 1 \mathrm{H}), 2.89-2.94(\mathrm{bs}, 1 \mathrm{H}), 2.37$ $(\mathrm{d}, J 2.2 \mathrm{~Hz}, 1 \mathrm{H}), 2.02-2.15(\mathrm{~m}, 1 \mathrm{H}), 1.86-1.97(\mathrm{~m}, 1 \mathrm{H}) .{ }^{13} \mathrm{C} \mathrm{NMR}:\left(\mathrm{CDCl}_{3}, 75 \mathrm{MHz}\right): \delta 137.65$, 128.30, 127.63, 127.59, 126.82, 84.23, 73.17, 72.83, 67.23, 64.92, 60.74, 36.51, 29.55. EI-MS: $m / z: 189$ [M-H]. 
(S)-[(3-Methoxypent-4-ynyloxy)methyl]benzene (9). To a suspension of $\mathrm{NaH}(1.26 \mathrm{mg}, 3.2$ $\mathrm{mmol})$ in THF $(10 \mathrm{~mL})$, was added alcohol $17(300 \mathrm{mg}, 1.578 \mathrm{mmol})$ at $0{ }^{\circ} \mathrm{C}$. After stirring for $20 \mathrm{~min}, \mathrm{MeI}$ (448 mg, $3.15 \mathrm{mmol}$ ) was added slowly and the resulting mixture was stirred for a further $2 \mathrm{~h}$. After completion, the reaction was quenched with ice-cold $\mathrm{H}_{2} \mathrm{O}(10 \mathrm{~mL})$ and extracted with EtOAc $(3 \times 10 \mathrm{~mL})$. The combined organic layers were dried over anhydrous $\mathrm{Na}_{2} \mathrm{SO}_{4}$, the solvent was evaporated under reduced pressure, and the residue was purified by silica gel column chromatography (EtOAc/hexanes, 1:9) to afford compound 9 as a yellow oily liquid (275 mg, 85\%).

$[\alpha]_{\mathrm{D}}{ }^{27}+4.2\left(c, 0.5, \mathrm{CHCl}_{3}\right) . \mathrm{IR}$ (neat) $v_{\max }: 3292,3030,2931,2863,2106,1728,1454,1362$, 1108, 1028, 739, $696 \mathrm{~cm}^{-1}$. ${ }^{1} \mathrm{H}$ NMR: $\left(\mathrm{CDCl}_{3}, 300 \mathrm{MHz}\right): \delta 7.20-7.33(\mathrm{~m}, 5 \mathrm{H}), 4.48(\mathrm{~s}, 2 \mathrm{H}), 4.12$ $(\mathrm{td}, J 2.2,6.0 \mathrm{~Hz}, 1 \mathrm{H}), 3.58(\mathrm{td}, J 2.2,6.0 \mathrm{~Hz}, 2 \mathrm{H}), 3.38(\mathrm{~s}, 3 \mathrm{H}), 2.35(\mathrm{~d}, J 2.26 \mathrm{~Hz}, 1 \mathrm{H}), 1.88-$ $2.08(\mathrm{~m}, 2 \mathrm{H}) ;{ }^{13} \mathrm{C} \mathrm{NMR:}\left(\mathrm{CDCl}_{3}, 75 \mathrm{MHz}\right): \delta 138.2,128.1,127.7,127.4,82.2,73.8,72.8,67.9$, 65.9, 56.3, 35.7; ESI-MS: $m / z$ : 227[M+Na]. HRMS calcd for $\mathrm{C}_{13} \mathrm{H}_{16} \mathrm{O}_{2} \mathrm{Na}$ 227.1047, found 227.1048 .

(2S,6S)-8-(Benzyloxy)-6-methoxyoct-4-yn-2-ol (8). $n$-BuLi (1.6 M in hexanes, $1.22 \mathrm{~mL}, 1.83$ $\mathrm{mmol})$ was added dropwise to a solution of $9(250 \mathrm{mg}, 1.22 \mathrm{mmol})$ in anhydrous THF (20 mL) at $-78{ }^{\circ} \mathrm{C}$ under $\mathrm{N}_{2}$ atmosphere. The mixture was allowed to stir for $30 \mathrm{~min}$ and then treated with $\mathrm{BF}_{3} \cdot \mathrm{OEt}_{2}(270 \mathrm{mg}, 1.83 \mathrm{mmol})$. After $10 \mathrm{~min}$, a solution of $(S)$-propylene oxide $(\mathbf{1 0}, 142 \mathrm{mg}$, $2.45 \mathrm{mmol})$ in anhydrous THF $(5 \mathrm{~mL})$ was added and the mixture was allowed to stir for $3 \mathrm{~h}$ at $-78{ }^{\circ} \mathrm{C}$. The resulting mixture was quenched with a mixture of $\mathrm{NaHCO}_{3}(10 \mathrm{~mL})$ and $\mathrm{NH}_{4} \mathrm{Cl}(10$ $\mathrm{mL})$ solutions at $-78{ }^{\circ} \mathrm{C}$. The mixture was then allowed to warm to room temperature and extracted with EtOAc $(3 \times 20 \mathrm{~mL})$, washed with $\mathrm{H}_{2} \mathrm{O}(10 \mathrm{~mL})$ and dried over $\mathrm{Na}_{2} \mathrm{SO}_{4}$. Removal of the solvent followed by purification on silica gel column chromatography (EtOAc/hexanes, 3:7) afforded compound 8 as colourless oil (220 mg, 65\%).

$[\alpha]_{\mathrm{D}}^{27}+21.3\left(c, 0.5, \mathrm{CHCl}_{3}\right)$. IR (neat) $v_{\max }: 3422,3030,2967,2930,2867,2196,1726,1453$, 1367, 1206, 1107, 940, 742, $699 \mathrm{~cm}^{-1} .{ }^{1} \mathrm{H}$ NMR: $\left(\mathrm{CDCl}_{3}, 500 \mathrm{MHz}\right): \delta 7.21-7.33(\mathrm{~m}, 5 \mathrm{H}), 4.47$ $(\mathrm{s}, 2 \mathrm{H}), 4.10(\mathrm{t}, J 6.76 \mathrm{~Hz}, 1 \mathrm{H}), 3.85-3.91(\mathrm{~m}, 1 \mathrm{H}), 3.57(\mathrm{t}, J 5.79 \mathrm{~Hz}, 2 \mathrm{H}), 3.35(\mathrm{~s}, 3 \mathrm{H}), 2.28-$ $2.41(\mathrm{~m}, 2 \mathrm{H}), 1.86-2.03(\mathrm{~m}, 2 \mathrm{H}), 1.23(\mathrm{~d}, J 6.7 \mathrm{~Hz}, 3 \mathrm{H}) .{ }^{13} \mathrm{C} \mathrm{NMR}\left(\mathrm{CDCl}_{3}, 75 \mathrm{MHz}\right): \delta 138.2$, $128.2,127.5,127.5,82.7,81.0,74.4,74.0,72.9,68.5,66.2,66.2,56.3,36.0,29.1,22.2$. ESI-MS: $\mathrm{m} / \mathrm{z}: 263[\mathrm{M}+\mathrm{H}]$. HRMS cacld for $\mathrm{C}_{16} \mathrm{H}_{24} \mathrm{O}_{3} 263.1647$, found 263.1657.

(2S,6S)-8-(Benzyloxy)-6-methoxyoct-4-yn-2-yl(3,5-dimethoxyphenyl)acetate (18). To a stirred solution of $8(150 \mathrm{mg}, 0.56 \mathrm{mmol})$ in $\mathrm{CH}_{2} \mathrm{Cl}_{2}(10 \mathrm{ml})$ at $0{ }^{\circ} \mathrm{C}$ was added $\mathrm{DCC}(232 \mathrm{mg} \mathrm{g}$, $1.12 \mathrm{mmol}$ ), followed by a catalytic amount of DMAP. After $5 \mathrm{~min}, 3,5$-dimethoxybenzeneacetic acid (121 mg, $0.62 \mathrm{mmol}$ ) was added and the resulting mixture was stirred for $1 \mathrm{~h}$ at room temperature. Upon completion, the mixture was quenched with water $(10 \mathrm{~mL})$ and then extracted with $\mathrm{CH}_{2} \mathrm{Cl}_{2}(20 \mathrm{~mL})$. The organic layer was washed successively with $10 \%$ aq. $\mathrm{HCl}$ solution followed by sat. $\mathrm{NaHCO}_{3}$ solution and brine. The organic layer was dried over $\mathrm{Na}_{2} \mathrm{SO}_{4}$, and concentrated in vacuo and the residue was purified by column chromatography (AcOEt / hexanes, 2:8) to afford compound 18 (200 mg, 80\%) as a colourless oil (200 mg, 80\%). 
$[\alpha]_{\mathrm{D}}^{27}-16.6\left(c, 0.5, \mathrm{CHCl}_{3}\right)$. IR (neat) $v_{\max }: 3448,2936,2130,1722,1600,1462,1290,1203$, 1154, 1107, 1065, 960, 837, 744, $695 \mathrm{~cm}^{-1} .{ }^{1} \mathrm{H}$ NMR: $\left(\mathrm{CDCl}_{3}, 500 \mathrm{MHz}\right): \delta$ 7.17-7.30 (m, 5H), $6.37(\mathrm{~d}, J 6.7 \mathrm{~Hz}, 2 \mathrm{H}), 6.29(\mathrm{~s}, 1 \mathrm{H}), 4.90-5.04(\mathrm{~m}, 1 \mathrm{H}), 4.42(\mathrm{~s}, 2 \mathrm{H}) 3.94-4.08(\mathrm{~m}, 2 \mathrm{H}), 3.71$ (s, 9H), 3.43-3.56 (m, 5H), $2.35-2.47(\mathrm{~m}, 2 \mathrm{H}), 1.27$ (d, J 5.79 Hz, 3H), $1.15(\mathrm{~d}, J 6.76 \mathrm{~Hz}, 2 \mathrm{H})$.

${ }^{13} \mathrm{C} \mathrm{NMR}:\left(\mathrm{CDCl}_{3}, 75 \mathrm{MHz}\right): \delta 170.5,160.6,138.3,135.9,128.2,127.5,107.1,99.0,81.5,80.7$, 72.8, 70.3, 69.7, 68.3, 66.2, 56.2, 25.6, 19.0. ESI-MS: $m / z: 458$ [M+NH 4 . HRMS calcd for $\mathrm{C}_{26} \mathrm{H}_{36} \mathrm{NO}_{6} 458.2542\left[\mathrm{M}+\mathrm{NH}_{4}\right]$, found 458.2558 .

(2S,6R)-8-Hydroxy-6-methoxyoctan-2-yl (3,5-dimethoxyphenyl)acetate (19). To a stirred solution of $18(200 \mathrm{mg}, 0.45 \mathrm{mmol})$ in EtOAc $(10 \mathrm{~mL}), 10 \% \mathrm{Pd} / \mathrm{C}(25 \mathrm{mg})$ was added and the mixture was stirred under $\mathrm{H}_{2}$ atmosphere at room temperature for $12 \mathrm{~h}$. After complete conversion, the mixture was filtered through Celite and the filtrate was concentrated under reduced pressure and then by purified by silica gel column chromatography (EtOAc/hexanes, 6:4) to afford compound 19 as an oily liquid (152 $\mathrm{mg}, 70 \%)$.

$[\alpha]_{\mathrm{D}}{ }^{27}-0.9\left(c, 0.5, \mathrm{CHCl}_{3}\right)$. IR (neat) $v_{\max }: 3327,2923,2849,1702,1612,1463,1417,1334$, 1294, 1238, 1205, 1152, 1062, 915, 818, 735, $654 \mathrm{~cm}^{-1} .{ }^{1} \mathrm{H} \mathrm{NMR}:\left(\mathrm{CDCl}_{3}, 500 \mathrm{MHz}\right): \delta 6.38(\mathrm{~d}$, $J 6.7 \mathrm{~Hz}, 2 \mathrm{H}), 6.28(\mathrm{~m}, 1 \mathrm{H}), 4.83-4.94(\mathrm{~m}, 1 \mathrm{H}), 3.76(\mathrm{~s}, 9 \mathrm{H}), 3.65-3.74(\mathrm{~m}, 2 \mathrm{H}), 3.52(\mathrm{~s}, 1 \mathrm{H})$, $3.47(\mathrm{~s}, 1 \mathrm{H}), 3.30(\mathrm{~s}, 2 \mathrm{H}), 1.24-1.64(\mathrm{~m}, 8 \mathrm{H}), 1.21(\mathrm{~d}, J 6.2 \mathrm{~Hz}, 3 \mathrm{H})$. ESI-MS: $m / z: 458$ $\left[\mathrm{M}+\mathrm{NH}_{4}\right]$. HRMS calcd for $\mathrm{C}_{21} \mathrm{H}_{25} \mathrm{NO}_{6} \mathrm{Na} 410.1575$, found 410.1579 .

(3R,7S)-7-[2-(3,5-Dimethoxyphenyl)acetoxy]-3-methoxyoctanoic acid (6): Compound 19 (15 $\mathrm{mg}, 0.38 \mathrm{mmol}$ ) was taken in $5 \mathrm{~mL}$ of distilled acetone at $0{ }^{\circ} \mathrm{C}$. Then a freshly prepared Jones reagent was added slowly at the same temperature until the orange brown color persists. The mixture was allowed to attain room temperature and the stirring was continued for further 30 min. The mixture was then quenched with water and extracted with EtOAc. Purification by silica gel column chromatography gave the acid 6 (100 mg, 80\%).

$[\alpha]_{\mathrm{D}}{ }^{27}+9.1\left(c, 0.8, \mathrm{CHCl}_{3}\right)$. IR (neat) $v_{\max }: 3447,2928,1721,1596,1458,1301,1267,1198$, $1165,1035,765,637 \mathrm{~cm}^{-1} .{ }^{1} \mathrm{H}$ NMR $\left(\mathrm{CDCl}_{3}, 500 \mathrm{MHz}\right): \delta 6.75(\mathrm{~s}, 2 \mathrm{H}), 6.30(\mathrm{~s}, 1 \mathrm{H}), 4.81-4.94$ $(\mathrm{m}, 1 \mathrm{H}), 3.78(\mathrm{~s}, 6 \mathrm{H}), 3.49(\mathrm{~m}, 1 \mathrm{H}), 3.45(\mathrm{~s}, 2 \mathrm{H}), 3.30(\mathrm{~s}, 3 \mathrm{H}), 2.47$ (dd, $J 15.7,6.3 \mathrm{~Hz}, 1 \mathrm{H}), 2.35$ $(\mathrm{dd}, J 15.7,5.3 \mathrm{~Hz}, 1 \mathrm{H}), 1.21-1.61(\mathrm{~m}, 6 \mathrm{H}), 1.98$ (d, $J 6.2 \mathrm{~Hz}, 3 \mathrm{H}) ;{ }^{13} \mathrm{C} \mathrm{NMR}:\left(\mathrm{CDCl}_{3}, 75 \mathrm{MHz}\right)$ : $\delta 175.4,171.2,170.2,159.9,137.2$, 107.2, 99.7, 78.3, 70.2, 55.9, 55.1, 41.7, 38.7, 35.3, 32.2. ESI-MS: $m / z$ : 286[M+NH 4 . HRMS calcd for $\mathrm{C}_{19} \mathrm{H}_{21} \mathrm{O}_{7} \mathrm{Na} 391.1732$, found 391.1714.

\section{(4S,8R)-8,11,13-Trimethoxy-4-methyl-4,5,6,7,8,9-hexahydro-1 $H$-benzo[d][1] oxacyclo-}

dodecine-2,10-dione (20). The compound $6(100 \mathrm{mg}, 0.27 \mathrm{mmol})$ was dissolved in $\mathrm{CF}_{3} \mathrm{COOH}$ $(7.5 \mathrm{~mL})$ and $\left(\mathrm{CF}_{3} \mathrm{CO}\right)_{2} \mathrm{O}(1.2 \mathrm{~mL})$. The solution was stirred overnight at room temperature and poured into an excess of $\mathrm{NaHCO}_{3}$ solution. The mixture was extracted with $\mathrm{Et}_{2} \mathrm{O}(3.5 \mathrm{~mL})$ and the extract was dried over anhydrous $\mathrm{Na}_{2} \mathrm{SO}_{4}$ and concentrated in vacuo and the residue was purified by column chromatography (EtOAc/hexanes, 1: 5) to afford compound $\mathbf{2 0}$ as a colorless oil (52 mg, 50\%).

$[\alpha]_{\mathrm{D}}{ }^{27}-16\left(c, 0.8, \mathrm{CHCl}_{3}\right)$. IR (neat) $v_{\max }: 3387,2936,1728,1659,1601,1457,1310,1271,1157$, 1084, 973, $681 \mathrm{~cm}^{-1} .{ }^{1} \mathrm{H} \mathrm{NMR}:\left(\mathrm{CDCl}_{3}, 500 \mathrm{MHz}\right): \delta 6.51(\mathrm{~s}, 1 \mathrm{H}), 6.42(\mathrm{~s}, 1 \mathrm{H}), 6.21(\mathrm{~d}, J 15.4$ $\mathrm{Hz}, 1 \mathrm{H}), 4.85$ (t, J $6.3 \mathrm{~Hz}, 1 \mathrm{H}), 3.81$ (s, 2H), 3.80 (s, 6H), 3.31 (d, J $18.6 \mathrm{~Hz}, 3 \mathrm{H},), 2.31$ (t, J 6.7 
$\mathrm{Hz}, 1 \mathrm{H}), 2.16(\mathrm{t}, J 6.7 \mathrm{~Hz}, 1 \mathrm{H}), 1.90-1.73(\mathrm{~m}, 2 \mathrm{H}), 1.51-1.36(\mathrm{~m}, 4 \mathrm{H}), 1.13$ (d, J $6.3 \mathrm{~Hz}, 3 \mathrm{H})$..

${ }^{13} \mathrm{C} \mathrm{NMR}\left(\mathrm{CDCl}_{3}, 75 \mathrm{MHz}\right): \delta 198.3,170.2,160.7,157.3,156.2,133.0,122.3,106.3,97.5,72.7$, 55.3, 55.1, 55.0, 39.3, 34.0, 39.9, 24.1, 20.1. ESI-MS: $m / z: 373$ [M+Na]. HRMS cacld for $\mathrm{C}_{19} \mathrm{H}_{26} \mathrm{O}_{6} \mathrm{Na} 373.1629$, found 373.1627.

(4S,8R)-11,13-Dihydroxy-8-methoxy-4-methyl-4,5,6,7,8,9-hexahydro-1H-benzo[d][1] $]$ oxacyclododecine-2,10-dione (5). To a stirred solution of iodine (1.1 g, $4.33 \mathrm{mmol})$ in dry benzene $(10 \mathrm{~mL})$ was added $\mathrm{Al}$ powder $(167 \mathrm{mg}, 4.70 \mathrm{mmol})$. The mixture was refluxed for $0.5 \mathrm{~h}$ and cooled to $10{ }^{\circ} \mathrm{C}$. To this mixture $\mathrm{Bu}_{4} \mathrm{NI}(253 \mathrm{mg})$ and a solution of compound 20 (50 mg, 0.14 mmol) in dry benzene $(4 \mathrm{~mL})$ were added. The mixture was stirred for $15 \mathrm{~min}$ at $10{ }^{\circ} \mathrm{C}$ and quenched with $2 \mathrm{M} \mathrm{HCl}$ at $0{ }^{\circ} \mathrm{C}$. The mixture was then extracted with ethyl acetate $(3 \times 20 \mathrm{~mL})$, the organic phase was washed with $\mathrm{NaHCO}_{3}$ solution followed by brine solution and dried over $\mathrm{Na}_{2} \mathrm{SO}_{4}$ and concentrated in vacuo. The residue was purified by coloumn chromatography (EtOAc/hexanes, $2: 1)$ gave the compound 5 as a colorless oil (30 mg, 65\%).

$[\alpha]_{\mathrm{D}}{ }^{27}-3.0(c, 1.0, \mathrm{EtOH})$. IR (neat) $v_{\max }: 3390,2921,2850,1709,1618,1532,1319,1258,1157$, 1028, 976, 841, 750, $643 \mathrm{~cm}^{-1}$; ${ }^{1} \mathrm{H}$ NMR: $\left(\mathrm{CDCl}_{3}, 300 \mathrm{MHz}\right): \delta 6.50(\mathrm{~s}, 1 \mathrm{H}), 6.19(\mathrm{~s}, 1 \mathrm{H}), 4.90(\mathrm{t}$, $J 6.2 \mathrm{~Hz}, 1 \mathrm{H}), 3.87$ (d, J 15.7 Hz, 1H), 3.71 (d, J 14.09 Hz, 1H), 3.57 (d, J 16.3 Hz, 1H), 3.30 (d, $J 5.1 \mathrm{~Hz}, 1 \mathrm{H}), 3.25(\mathrm{~s}, 3 \mathrm{H}), 3.12(\mathrm{dd}, J 14.11,8.1 \mathrm{~Hz}, 1 \mathrm{H}), 1.51-1.83(\mathrm{~m}, 6 \mathrm{H}), 1.21(\mathrm{~d}, J 5.1$ $\mathrm{Hz}, 3 \mathrm{H}) .{ }^{13} \mathrm{C} \mathrm{NMR},\left(\mathrm{CDCl}_{3}, 75 \mathrm{MHz}\right): \delta 170.7,160.2,157.3,133.0,117.2,112.4,101.7,73.9$, 72.1, 54.1, 49.2, 41.0, 31.2, 30.4, 19.2, 17.2. ESI-MS: $m / z: 345$ [M+Na]. HRMS calcd for $\mathrm{C}_{17} \mathrm{H}_{22} \mathrm{O}_{6} \mathrm{Na} 345.1307$, found 345.1311.

\section{Acknowledgements}

C.D.V. thanks CSIR, New Delhi for the award of a fellowship.

\section{References}

1. Kobayashi, A.; Hino, T.; Yata, S.; Itoh, T. J.; Sato, H.; Kawazu, K. Agric. Biol. Chem. 1988, 52.

2. Robeson, D. J.; Strobel, G. A. Z. Natuerforsch. Sect. C 1981, 36C, 1081.

3. Furstner, A.; Castneer, A. S. S.; Radkowski, K.; Lehmann, C. W. J. Org. Chem. 2003, 68, 1521, and references cited therein.

http://dx.doi.org/10.1021/jo026686q

4. He, J.; Wijeratne, E. M. K.; Bashyal, B. P.; Zhan, J.; Seliga, C. J.; Liu, M. X.; Pierson, E. E.; Pierson, L. S. III; Van Etten, H. D.; Gunatilaka, A. A. L. J. Nat. Prod. 2004, 67, 1985. http://dx.doi.org/10.1021/np040139d

5. Whitesell, L.; Bagatell, R.; Falsey, R. Current Cancer Drug Targets. 2003, 3, 349. http://dx.doi.org/10.2174/1568009033481787 
6. Liang, Q.; Sun, Y.; Yu. B.; She, X.; Pan, X. J. Org. Chem. 2007, 72, 9846. http://dx.doi.org/10.1021/jo701885n

7. Baker, P. M.; Bycroft, B. W.; Roberts, J. C. J. Chem, Soc. (C) 1967, 1913.

8. Liang, Q.; Zhang, J.; Quan, W.; Sun, Y.; She, X.; Pan. X. J. Org. Chem. 2007, 72, 2694. http://dx.doi.org/10.1021/jo070159v

9. Czernecki, S.; Georgoulis, C.; Provelenghiou, C.; Fusey, G. Tetrahedron Lett. 1976, 17, 3535 . http://dx.doi.org/10.1016/S0040-4039(00)71351-7

10. Kanai, K.; Sakamoto, I., Ogawa, S.; Suami, T. Bull. Chem. Soc. Jpn. 1987, 60, 1529. http://dx.doi.org/10.1246/bcsj.60.1529

11. Gao, Y.; Hanson, R. M.; Klunder, J. M.; Ko, S. Y.; Masamune, H.; Sharpless K. B.; J. Am. Chem. Soc. 1987, 109, 5765. http://dx.doi.org/10.1021/ja00253a032

12. Rama Rao, A. V.; Bhanu, M. N.; Sharma, G. V. M. Tetrahedron Lett. 1993, 34, 707. http://dx.doi.org/10.1016/S0040-4039(00)61612-X

13. Schaus, S. E.; Brandes, B. D.; Larrow, J. F.; Tokunaga, M. K. B.; Hansen. A. E.; Gould, M. E.; Jacobsen. E. N. J. Am. Chem. Soc. 2002, 124, 1307. http://dx.doi.org/10.1021/ja0167371

14. McNeill, A. H.; Thomas, E. J. Tetrahedron Lett. 1993, 34, 1669. http://dx.doi.org/10.1016/0040-4039(93)85037-W

15. Bowden, K.; Heilbron, M. I.; Jones, E. R. H.; Weedon, B. C. L. J. Chem. Soc. 1946, 39. http://dx.doi.org/10.1039/jr9460000039

16. Bowers, A. T.; Halsall, G.; Jones, E. R. H.; Lemin, A. J. J. Chem. Soc. 1953, 2548. http://dx.doi.org/10.1039/jr9530002548

17. Kreipl, A. T.; Reid, C.; Steglich, W. Org. Lett. 2002, 4, 3287. http://dx.doi.org/10.1021/o1026555b

18. Madhusudana Rao, J.; Reddy, G. V.; Chandra, R. S.; Babu, K. S. Tetrahedron Lett. 2009, 50, 4117. http://dx.doi.org/10.1016/j.tetlet.2009.04.120

19. Yadav, J. S.; Raju, A.; Ravindar, K.; Reddy, B. V. S. Synthesis 2010, 5, 797. http://dx.doi.org/10.1055/s-0029-1218621

20. Rajesh, K.; Rajesh, V.; Selvam, J. J. P.; Babu, D. C.; Venkateswarlu, Y. Helv. Chim. Acta 2010, 93, 147. http://dx.doi.org/10.1002/hlca.200900136 